analyse the outcome of patients diagnosed to have pancreatic cancer in clinical practice in North London.

Methods In this duel centre retrospective study, a years worth of pancreatic cancer diagnoses was compiled using the North London Cancer Network Multi-disciplinary team meeting data base. The patients records were then searched gathering information on their dates of diagnosis; referral to our hepatobiliary surgeons at a local tertiary referral centre; whether they had a pre-operative stent; the date of their surgery (if they survived long enough to have it) and they're ultimate outcome.

Results 68 patients within our sector received a diagnosis (histological/endoscopic/radiological) of pancreatic cancer over the course of 1 year (May 2010-May 2011). Of this cohort 20 (29.4\%) were referred for surgical opinion. During the lag between diagnosis and surgical review, 9 (45\%) patients received endoscopic biliary drainage and stent insertion (all were 1st pass). The total number to ultimately receive their Whipple's was 5 (25\%). In four patients in whom surgery was felt to be an option, aggressive disease and complications leading to a lengthy in patient stay at the point of diagnosis meant that the physical condition of the patient had deteriorated to the point where they were no longer fit for surgery/ inoperable. Only one patient proceeded straight to operation without prior stenting. Two patients had their operations privately. Unfortunately details of any post operative complications are not available.

Conclusion Our experiences of pancreatic cancer is that at the point of diagnosis most cancers were inoperable 48 of 68 (70\%). Within our study period only 5 of 68 (7\%) patients had surgery for pancreatic cancer. The majority of patients even when initially considered for surgery (75\%) do not end up having a resection. When patients are referred with symptoms of obstructive jaundice, knowing that the majority will not undergo surgery and also knowing in clinical practice that it is difficult to get surgical resection within 10 days of diagnosis, the humane thing to do instinctively is to stent and achieve biliary drainage. Achieving biliary drainage helps in improving the patients symptom profile and additionally allows chemotherapeutic options in those whose jaundice resolves.

Competing interests None declared.

\section{PM0-098 DIAGNOSTIC YIELD OF ERCP AND EUS FNA IN A TERTIARY PANCREATIC CANCER CENTRE}

\section{doi:10.1136/gutjnl-2012-302514b.98}

${ }^{1} \mathrm{D}$ McClements, ${ }^{*} \mathrm{H}$ Smart, ${ }^{2} \mathrm{P}$ Whelan. ${ }^{1}$ Department of Gastroenterology, Royal Liverpool University Hospital, Liverpool, UK; ${ }^{2}$ Department of Hepatobiliary, Royal Liverpool University Hospital, Liverpool, UK

Introduction Patients with suspected pancreatic malignancy on cross sectional imaging are often referred for endoscopic investigations with a view to obtaining a definitive histological diagnosis. We aimed to assess the diagnostic yield of brushings and biopsies taken at endoscopic retrograde cholangiopancreatography (ERCP) and endoscopic ultrasound with fine needle aspiration (EUS FNA) in patients with pancreatic malignancy proven by operative histology.

Methods A retrospective audit of 125 patients undergoing surgery for pancreatic malignancy at the Royal Liverpool University Hospital (RLUH) from January 2009 to December 2011 was carried out. Of these patients, 35 underwent investigation at RLUH and are included in this analysis, two of these required two investigations. Fifty patients had ERCP at an external Trust and 40 patients went straight to surgery without endoscopic intervention. Data were extracted from the pancreatic surgery database and electronic patient records for demographics and histology reports taken at the time of endoscopy and surgery.
Results Overall 123 patients had operative histology confirming pancreatic adenocarcinoma, two patients had neuroendocrine tumour (NET) of the pancreas. Of the 35 patients undergoing investigation at RLUH, 34 had pancreatic adenocarcinoma and one had NET of the pancreas. Fifteen of 29 (52\%) patients had brushings confirmatory of malignancy at ERCP, 9 of 29 (31\%) had no malignant cells seen on brushings at ERCP, 3 of 29 (10\%) had equivocal results suggestive but not diagnostic of malignancy. Brush cytology was not obtained in two patients, one patient suffered a perforation at ERCP requiring emergency surgery and one patient had failed cannulation of the CBD. Five of eight patients had an EUS FNA confirmatory of malignancy, two of eight had no malignant cells seen and 1 had equivocal results. In our cohort the sensitivity of ERCP alone is $56 \%$ (95\% CI 36\% to $74 \%$ ) the combined sensitivity for ERCP and EUS 57\% (95\% CI 40\% to 73\%).

Conclusion The sensitivity of brush cytology at ERCP for has previously been reported to be between $50 \%$ and $65 \%{ }^{1-3}$ for pancreatic malignancies. This is comparable to our findings in this cohort of patients with proven pancreatic malignancy. Positive histology from ERCP to EUS FNA can be confirmatory of pancreatic malignancy, but caution should be used when interpreting negative histology results given the intermediate sensitivity of these investigations.

Competing interests None declared.

\section{REFERENCES}

1. Mansfield JC, Griffin SM, Wadehra V, et al. A prospective evaluation of cytology from biliary strictures. Gut 1997; 40:671-7.

2. Macken E, et al. Brush cytology of ductal strictures during ERCP. Acta Gastroenterol Belg 2000;63:254-9.

3. Temino Lopez-Jurado $\mathbf{R}$, et al. Diagnostic yeild of brush cytology for biliary stenosis during ERCP. Rev Esp Enferm Digest 2009;101:385-94.

\section{PM0-099 A COMPARATIVE STUDY OF LAPAROSCOPIC VS OPEN DISTAL PANCREATECTOMY}

doi:10.1136/gutjnl-2012-302514b.99

D J Malde, ${ }^{*}$ Y Khaled, J Packer, N De Liguori, R Deshpande, D O’Reilly, D Sherlock, B J Ammori. The HPB Unit, North Manchester General Hospital, Manchester, UK

Introduction The laparoscopic approach to distal pancreatectomy for benign and malignant diseases appears to offer advantages and is replacing open surgery in some centres. However, well-designed studies comparing laparoscopic distal pancreatectomy (LDP) to open distal pancreatectomy (ODP) are limited. We present a single-institution study comparing the outcomes of LDP to ODP.

Methods The demographic details, clinical characteristics and outcomes of patients who underwent laparoscopic distal pancreatectomy were compared to those who had the surgery performed by open technique. The two approaches were compared on an intention-to-treat basis. Data shown represent medians.

Results Between 2002 and 2009, 32 patients (20 female) underwent 16 LDP and 16 ODP respectively. The laparoscopic and open groups were comparable for age ( 57 vs 63 years, $\mathrm{p}=0.584$ ), sex distribution and tumour size ( 3.9 vs $4 \mathrm{~cm}, \mathrm{p}=0.939)$. Both groups had a comparable number of malignant cases ( $56 \%$ vs $50 \%, p=1.0$ ). Although LDP took longer to complete ( 287.5 vs $240 \mathrm{~min}, \mathrm{p}=0.061$ ), it was associated with significantly lower blood loss (300 vs $500 \mathrm{ml}$, $p=0.031)$ but comparable perioperative transfusion rate $(p=0.471)$. The laparoscopic approach was associated with a significantly higher spleen-preservation rate (overall: $50 \%$ vs $12.5 \%, \mathrm{p}=0.05$; benign pathology: $85.7 \%$ vs $25 \%, p=1.0)$. LDP patients had a significantly lower HDU stay ( 1 vs 4.5 days, $\mathrm{p}<0.001$ ) and a significantly lower postoperative hospital stay (6.5 vs 13.5 days, $p=0.001$ ). There was no significant difference in the postoperative morbidity and the $\mathrm{R} 0$ resection margin status. 
Conclusion The laparoscopic approach to distal pancreatectomy results in significantly lower blood loss, and shorter HDU and hospital stay compared with open surgery. The postoperative morbidity and $\mathrm{R} 0$ resection margin rates were comparably similar.

Competing interests None declared.

\section{PM0-100 OUTCOME OF MINIMALLY INVASIVE PANCREATIC NECROSECTOMY WITHOUT IRRIGATION}

doi:10.1136/gutjnl-2012-302514b.100

D J Malde, ${ }^{*}$ S S Raza, N Khan, A Aldouri, K Menon, A M Smith. The HPB Unit, St James University Hospital, Leeds, UK

Introduction Necrotizing Pancreatitis with secondary infection of the pancreatic tissue is associated with significant morbidity and mortality. Current evidence suggests that a minimally invasive retroperitoneal necrosectomy (MIRP) is feasible, well tolerated and beneficial for the patient when compared with open surgery.

Methods A total of 16 patients who underwent MIRP from September 2007 to April 2011 were included in the study. Current minimal access techniques all recommend routine irrigation but we aim to show that comparable results can be achieved without irrigation.

Results The mean age was 52.5 years with 13 patients transferred from other centres. The aetiology was gallstones (13), alcohol (1), idiopathic (1) and hyperlipidaemia (1). The average time before 1st necrosectomy was 50.2 days. The mean number of procedures was 3.3 (range $1-7$ ) with one patient requiring an open procedure. One patient required post-necrosectomy ICU admission. 13 patients had nasojejunal feed and four patients started with parenteral feed which was later converted to nasojejunal. Five patients developed a pancreatic fistula, three patients developed colonic fistula and two patients died. Mean inpatient stay was 82.6 days (range 31-182).

Conclusion This series suggests that doing MIRP without irrigation has results comparable to other centres carrying out routine irrigation.

Competing interests None declared.

\section{PM0-101 ENTERAL NUTRITION IN ACUTE PANCREATITIS: NASOGASTRIC OR NASOJEJUNAL?}

\section{doi:10.1136/gutjnl-2012-302514b.101}

D J Malde, ${ }^{*}$ A Suppiah, T Arab, K Menon, A M Smith. The HPB Unit, St James University Hospital, Leeds, UK

Introduction Enteral feeding is beneficial in patients with severe acute pancreatitis. A published series suggests $100 \%$ can be fed via the nasogastic (NG) route.

Methods 146 consecutive patients (January-December 2010) admitted with acute pancreatitis (AP) were reviewed to assess the safety and tolerance of $\mathrm{NG}$ feed. In all severe AP patients nutrition was initially delivered via an NG tube, if they were not absorbing a nasojejunal (NJ) tube was inserted.

Results 29 patients were identified as having poor outcome. 127 (87\%) patients were able to commence oral intake within $72 \mathrm{~h}$ of admission. $19(13 \%)$ patients required additional enteral or parenteral nutritional support. 16 patients were commenced on NG feed but two patients needed conversion to $\mathrm{NJ}$ feed. Three patients were directly commenced on NJ feed but one needed conversion to parenteral feed. Only one patient had been commenced on parenteral feed prior to transfer. Need for nutritional support was a significant indicator of poor outcome; morbidity $13 / 19$ vs $12 / 127$ $(p<0.0001)$ and mortality $6 / 19$ vs $1 / 127(p<0.0001)$.
Conclusion Nasogastric feeding is well tolerated in the majority (73.7\%) of patients with severe AP. NG feeding should be first line, but if failing a rapid change to the NJ route instituted.

Competing interests None declared.

\section{PM0-102 MEMBRANOUS EXPRESSION OF SULFATASE-2 IS ASSOCIATED WITH A POORER PROGNOSIS IN PATIENTS FOLLOWING PANCREATIC CANCER RESECTION}

doi:10.1136/gutjnl-2012-302514b.102

${ }^{1} \mathrm{D}$ Televantou, ${ }^{*}{ }^{1,2} \mathrm{~K}$ Y D Hui, ${ }^{3} \mathrm{R}$ Lochan, ${ }^{3} \mathrm{R}$ Charnley, ${ }^{4} \mathrm{~A} \mathrm{D}$ Burt, ${ }^{1,5} \mathrm{H}$ L Reeves. ${ }^{1}$ Northern Institute for Cancer Research, Newcastle University, Newcastle upon Tyne, UK; ${ }^{2}$ Department of HPB and Transplant Surgery, Freeman Hospital, Newcastle upon Tyne, UK; ${ }^{3}$ Department of Surgery, Freeman Hospital, Newcastle upon Tyne, UK; ${ }^{4}$ Institute of Cellular Medicine, Newcastle University, Newcastle upon Tyne, UK; ${ }^{5}$ Department of Hepatology, Freeman Hospital, Newcastle Upon Tyne, UK

Introduction Pancreatic adenocarcinomas are resistant to medical therapies and associated with a poor prognosis. Sulfatase 2 (SULF2) is one of two extracellular heparan sulphate 6-O-endosulfatases that modulate ligand activated FGF and Wnt signalling. SULF2 expression is dramatically upregulated at mRNA levels in pancreatic cancers (NCBI GEO). We have investigated SULF2 protein expression in pancreatic adenocarcinomas, in association with clinicopathological parameters.

Methods Immunohistochemistry for SULF2 was performed on archived FFPE (Formalin-Fixed paraffin Embedded) blocks from 21 resected primary pancreatic adenocarcinomas, most of which were histologically defined as ductal (19/21, 90.5\%). Membranous and cytoplasmic expression of SULF2 in tumour and stromal cells were separately assessed. Additionally, immunostaining for $\boldsymbol{\alpha}$-Smooth Muscle Actin (a-SMA) was performed for further cell characterisation. Results SULF2 was expressed in tumour cells in the majority of the tumours $(18 / 21,86 \%)$. This expression was either cytoplasmic (13/ $21,61.9 \%)$, membranous $(12 / 21,57.1 \%)$ or both $(17 / 21,80.9 \%)$. Membranous positivity was found almost exclusively in tumours with low differentiated areas $(11 / 12, p=0.007)$. Membranous overexpression was also associated with shorter patient survival $(p=0.011)$. Spindle-shaped cells of desmoplastic tumour stroma showed strong cytoplasmic positivity in all tumours studied $(21 / 21$, $100 \%$ ). These cells were also positive for a-SMA, a marker of activated pancreatic stellate cells. Non-neoplastic pancreas showed only focal positivity for SULF2; this involved mainly endothelial, and scattered epithelial cells of exocrine pancreas.

Conclusion SULF2 over- expression is common in pancreatic adenocarcinomas, in both the ductal cancer cells as well as the desmoplastic tumour stroma. Tumour cell membranous localisation and over expression is associated with a more aggressive tumour behaviour and poorer patient survival. SULF2 is a novel candidate biomarker in patients for pancreatic cancer, identifying those with a poorer prognosis, as well as those who may benefit from therapies inhibiting SULF2.

Competing interests None declared.

\section{PMO-103 PROGNOSTIC VALUE OF POST OPERATIVE CA19-9 IN PATIENTS UNDERGOING PANCREATICODUODENECTOMY FOR PANCREATIC ADENOCARCINOMA}

doi:10.1136/gutjnl-2012-302514b.103

D J Malde, E Jeans, ${ }^{*}$ N De Liguori, R Deshpande, B J Ammori, D J Sherlock, D O'Reilly. The HPB Unit, North Manchester General Hospital, Manchester, UK

Introduction Pancreatic Adenocarcinoma accounts for over $90 \%$ of Pancreatic malignancy with overall survival being $<5 \%$ at 5 years. CA $19-9$ is a commonly used tumour marker with levels in excess of $200 \mathrm{U} / \mathrm{ml}$ being $90 \%$ sensitive for pancreatic malignancy. Pre- 